\title{
Ärztliche Vorsorgeuntersuchungen an Schweizer Flughäfen
}

\section{S. Icher \\ F. Liegen}

Im Auftrag der Arbeitsgemeinschaft Schweizer Fachärzte für Gute Bildqualität (ASFGB)

\begin{abstract}
Flugpassagiere müssen sich auch in der Schweiz auf schärfere Sicherheitskontrollen einstellen. Die meisten Unannehmlichkeiten werden akzeptiert, doch gegen den Einsatz von Körperscannern regt sich seit geraumer Zeit der Widerstand. Kritisiert wird vor allem, dass die erzeugten Bilder die Intimsphäre verletzen und Datenschutzregelungen unterlaufen. Denn neben Plastiksprengstoff sind auch die «körperlichen Problemzonen» sichtbar.
\end{abstract}

\section{Gegen den Einsatz von Körperscannern regt sich}

\section{seit geraumer Zeit Widerstand}

Dass sich die Bildgebung hingegen auch diagnostisch nutzen lassen könnte, hat die Schweizerische Ärzteschaft frühzeitig erkannt und die FMH entsprechend mobilisiert, in Verhandlungen mit dem Bundesamt für Zivilluftfahrt (BAZL) einzutreten. Zunächst verliefen diese Verhandlungen zäh, doch die seitens der Ärzteschaft eingebrachten neuen Aspekte und Argumente konnten das BAZL letztendlich überzeugen. Inzwischen wurden sogar

\section{Abbildung 1}

Aufnahmen eines Körperscanners. Wegen der schlechten Bildauflösung raten Schweizer Ärzte von diesem Verfahren ab.
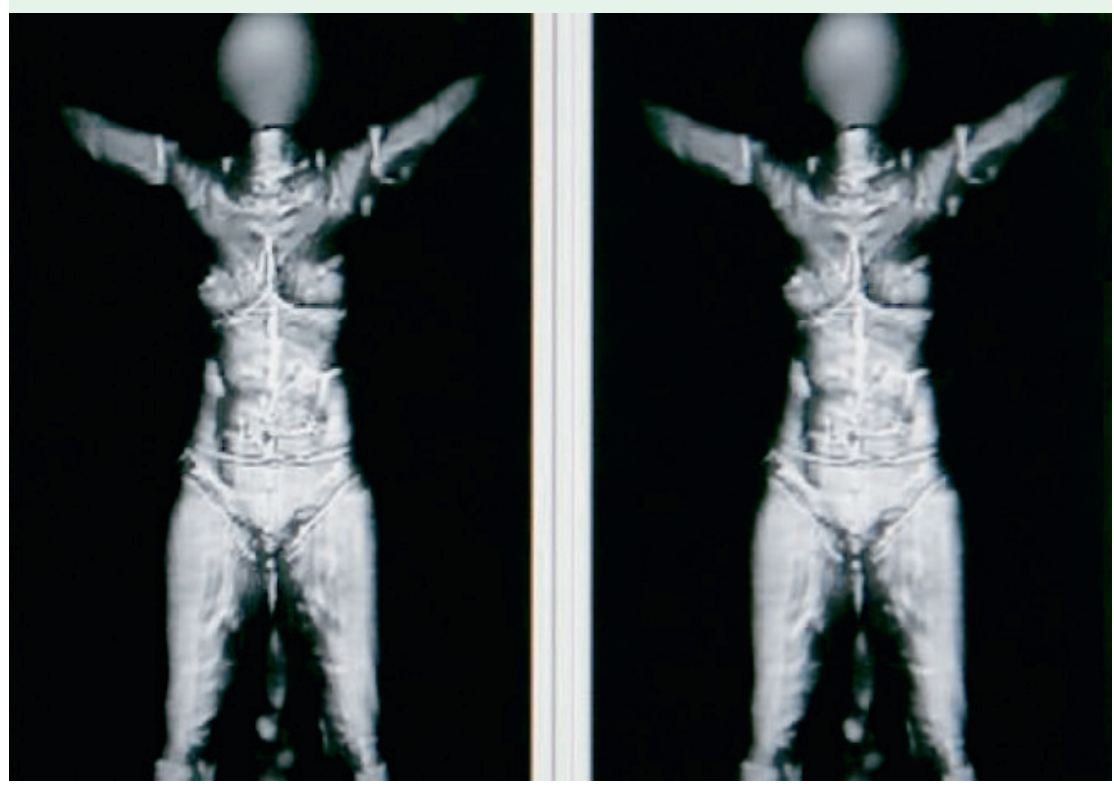

konkrete Massnahmen beschlossen, die die Widerstände gegen die Einführung bildgebender Verfahren an Schweizer Flughäfen voraussichtlich ganz ausräumen werden.

Ab dem 1. April 2010 sollen die Kontrollen an Schweizer Flughäfen von Schweizer Fachärzten durchgeführt werden. Da diese der ärztlichen Schweigepflicht unterliegen, sind Bedenken wegen eines möglicherweise unzureichenden Datenschutzes nicht mehr angebracht. Und die heimelige Atmosphäre von - wenn auch kleinen - Untersuchungskabinen neben dem Laufband für das Handgepäck gewährleistet einen ausreichenden Schutz der Privatsphäre.

Es gibt zudem wesentliche Änderungen der bisherigen Pläne des BAZL: Statt der Millimeter-WaveScanner, wie sie zum Beispiel auf dem Amsterdamer Flughafen Schiphol eingesetzt werden, kommt in der Schweiz die Ganzkörper-Sonographie zur Anwendung, die durch verschiedene Spezialisten durchgeführt wird. Angaben über Alter, Geschlecht, Vorerkrankungen usw. - kodiert auf dem Magnetstreifen der Bordkarte - dienen der gezielten Zuweisung zu einem Internisten, Gynäkologen, Urologen oder Pädiater. Der Schwerpunkt liegt verständlicherweise auf der abdominellen Sonographie. Ein ausreichender Füllungszustand der Harnblase - idealerweise durch Getränke erzeugt, die die Passagiere ohnehin nicht mit ins Flugzeug nehmen dürfen erlaubt bei Männern auch die Darstellung der Prostata. Ein anderes Beispiel sind Verlaufsbeurteilungen einer Schwangerschaft. Zukünftig soll daher auch nicht mehr von Sicherheitskontrollen die Rede sein, sondern von ärztlichen Vorsorgeuntersuchungen zur kurz- und langfristigen Sicherung der Gesundheit.

Im Gegensatz zu den Körperscannern sind Ultraschalluntersuchungen grundsätzlich auch in der Lage, potentiell gefährdende Gegenstände in Körperöffnungen zu erkennen. Digitale, gegebenenfalls endoskopische Anschlussuntersuchungen durch die Spezialisten für die jeweilige Körperöffnung zerstreuen letzte Zweifel sowohl in Bezug auf die Flugsicherheit als auch die Differentialdiagnose. Ein weiterer Vorteil der neuen Regelungen: Die ärztlichen Vorsorgeleistungen sind bereits mit den Kosten für das Flugticket abgegolten (sogenannter Kerosin- und Facharztzuschlag) und entlasten die Krankenkassen. 
Für Passagiere der Business Class besteht darüber hinaus die Möglichkeit, einen noch umfassenderen Gesundheits-Check durchführen zu lassen (u.a. grosses Labor, Belastungs-EKG). Dafür rechtfertigt sich der Einsatz von entsprechend qualifiziertem ärztlichem Fachpersonal. Eine renommierte Schweizer Universitätsklinik hat denn auch bereits zugesagt, ihre Leitenden Ärzte in angemessenem Rahmen dafür freizustellen. Für First-Class-Passagiere sollen sich sogar Klinikdirektoren gemeldet haben.

\section{Abbildung 2}

Die Interpretation der Bilder ist mitunter schwierig: Darstellung eines dislozierten Herzschrittmachers durch den Körperscanner.

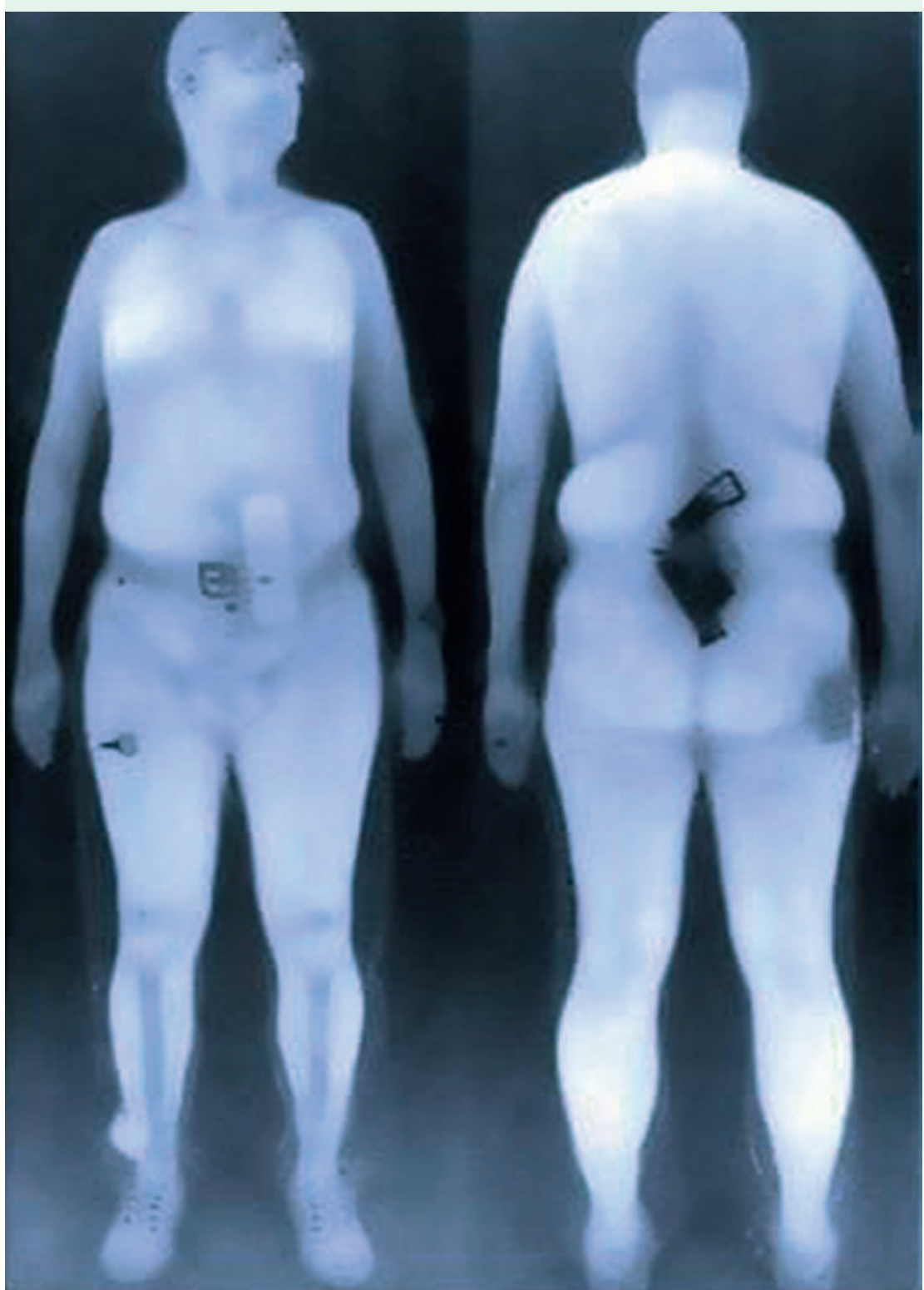

Viele Fluggesellschaften bieten auch den Teilnehmern von Vielfliegerprogrammen an, gesammelte Flugmeilen gegen ärztliche Leistungen einzulösen. Vielflieger kommen zudem in den ständigen Genuss einer second opinion.

Passagiere, die Zusatzleistungen in Anspruch nehmen, können sich ihre Fachärztin oder ihren Facharzt selbst aussuchen. Um Flugpassagieren die Auswahl zu erleichtern, dürfen die Fachärzte zwar nicht direkt für sich werben, jedoch auf sich aufmerksam machen - so zum Beispiel die Gynäkologen mit «Abstriche an der Zervix, aber nicht an der Gesundheit!» oder die Urologen mit «Wer häufig auf die Toilette muss, hat nicht nur im Flugzeug ein Problem!»

\section{Vielflieger können gesammelte Flugmeilen gegen ärztliche Leistungen eintauschen}

Die zuletzt genannte Warnung der Urologen deutet bereits an, dass diese mit den zukünftigen Massnahmen nicht rundum zufrieden sind. Sie wehren sich nämlich gegen Pläne des BAZL, Toilettengänge eine Stunde vor der Landung des Flugzeugs zu untersagen. Originalton SGU: «Es wird so viel über die Körperscanner geredet, dabei ist doch das Toilettenverbot der eigentliche Skandal der geplanten Sicherheitsbestimmungen. Wir treten vehement für die Rechte zahlreicher Passagiere mit imperativem Harndrang ein.»

\section{Abbildung 3}

Sonographischer Querschnitt durch die Prostata bald ein alltägliches Bild auf Schweizer Flughäfen?

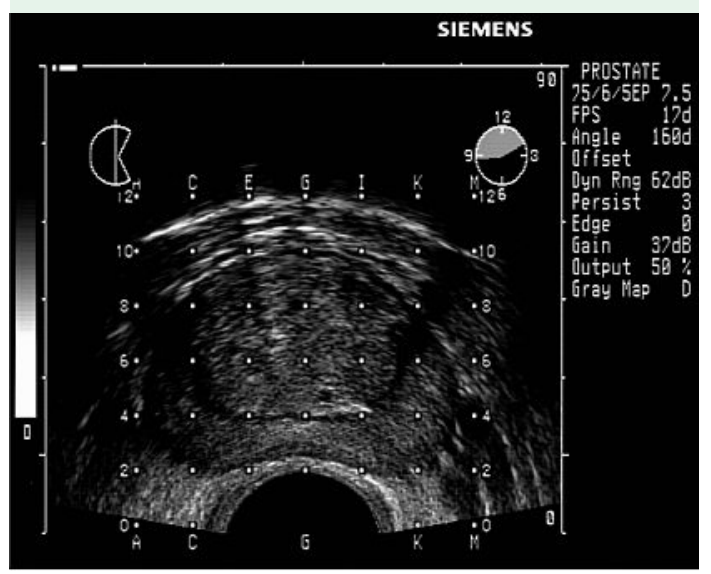

